\title{
SOME RECENT APPROACHES TO SMALLER, FASTER DEVICES FOR REMOTE PET
}

\section{RADIOLABELING.}

D.M. Jewett, T.J. Mangner, G.K. Mulholland, S.A. Toorongian, A.C. Sutorik, G.L. Watkins, M.R. Kilbourn

Division of Nuclear Medicine, University of Michigan Medical School, Ann Arbor, MI 48109-0552

No-carrier-added PET radiolabels involve submicrogram amounts of material. Adaptation of sample handling techniques already developed for analytical HPLC and GLC on this same scale has several important consequences for PET chemistry. First, a large variety of chromatographic equipment, associated techniques and experience become directly available for the construction of remote radiolabeling apparatus, facilitating automatic control and lowering costs. Secondly, operations based on trap-and-purge in a flowing process stream, and reactions in columns rather than discrete vessels are inherently faster. Columns are suited to rapid recycle, and can be disposable in many cases. To the extent that the radiolabel can be maintained in a volume of $<100$ ul throughout the process, final chromatography for simultaneous assessment of chemical and radiochemical purity as well as for purification is simplified. Lastly, the whole procedure may be accomplished in a small shielded bench-top apparatus, saving space and allowing greater flexibility of operations.

In order to exploit the inherent advantages of miniature flow systems, we have developed the captive solvent approach as a general way of accomplishing reactions in small disposable or recyclable columns. $(1,2)$ In this method a substrate dissolved in an appropriate solvent is trapped within a porous matrix in a column, allowing efficient phase transfer of a radiolabel from a gas or liquid phase. The captive solvent approach has been applied to the syntheses of $\left[{ }^{11} \mathrm{C}\right]$ acetate, $\left[{ }^{1} 1_{\mathrm{C}} \mathrm{C}\right.$ palmitate $(1),\left[{ }^{1} \mathrm{C}\right]$ methyl bromide, [11 C]benzodiazepines (2), [150]butanol (3) and to the activation of [18F]fluoride ion. An increasing number of fibrous or microporous polymers are becoming available, allowing the adaptation of this approach to a variety of reaction conditions. Trap-and-purge methods have been adapted from both GLC and HPLC techniques both for concentrating gas-phase reactants and for HPLC injections. These permit refocusing the radioactivity at various points within a system to facilitate miniaturization and final purification by HPLC.

To realize fully the advantages of miniature flow systems new methods for rapid cooling and heating are required. A new generation of reliable, inexpensive temperature controllers allows precise control and monitoring of temperature at many points in a system. Controlled-temperature heat guns allow fast, precise heating on demand. Where applicable, positive temperature coefficient heating devices save space, since no controllers are needed. Small metal heating blocks replace ovens for catalytic reactors up to $400^{\circ}$. Heating blocks can be used with pneumatic clamping devices for rapid heating and subsequent cooling. Even faster heating rates can be achieved if heating elements are contained within the flow paths, as exemplified by internally heated transfer lines and novel trap-and-purge devices for concentrating $[11 \mathrm{C}] \mathrm{CO}_{2}$. (4) Vortex tubes allow rapid cooling with only 
compressed air. A single device incorporating both a heat gun and vortex tube allows rapid heating and cooling over the range -330 to $+200^{\circ}$. Simple techniques for handling liquid $\mathrm{CO}_{2}$ allow rapid cooling to about $-70^{\circ}$. Ice reservoirs or air jackets eliminate the need for flowing cooling water.

Development and routine operation of PET radiosyntheses are facilititated by the detection of movement of radioactivity at several points in a system. We have adapted a variety of miniature flow ionization (5) detectors for this purpose. Where applicable, inexpensive flow detectors based on PIN photodiodes (6) allow selective detection of positrons in the presence of high background levels of gamma radiation. Simple chargeintegrating amplifiers are used instead of digital counting circuitry, permitting ready interfacing with LCD displays.

1. Jewett, D.M., Ehrenkaufer, R.L. and Ram, S., Int. J. Appl. Radiat. Isot. $\underline{36}$, 672 (1985).

2. Watkins, G.L., Jewett, D.M., Mulholland, G.K., Kilbourn, M.R. and Toorongian, S.A., Int. J. Appl. Radiat. Isot., in press.

3. Takahashi, K., Murakami, M., Hagami, E., Sasaki, H., Kondo, Y., Mizusawa, S., Nakamichi, H., Iida, H., Miura, S., Kanno, I., Uemura, K. and Ido, T., In Proceedings Sixth International Symposium on Radiopharmaceutical Chemistry, Boston, 1986, p.81.

4. Jewett, D.M., Rev. Sci. Instrum. 58, 1964 (1987).

5. Sipila, H.T., Heselius, S.J., Saarni, H.K. and Ahlfors, T., Nucl. Instr. Meth. A238, 542 (1985).

6. Hichwa, R.D. and Tsang, C., J. Label. Comp. Radiopharm. 19, 1346 (1982). 